\title{
The low-energy limit of validity of the intranuclear cascade model
}

\author{
J. Cugnon ${ }^{\mathrm{a}}$ and P. Henrotte ${ }^{\mathrm{b}}$ \\ University of Liège, Institute of Physics B5, Sart Tilman, B-4000 Liège 1, Belgium
}

Received: 2 September 2002 /

Published online: 6 March 2003 - (C) Società Italiana di Fisica / Springer-Verlag 2003

Communicated by G. Orlandini

\begin{abstract}
The intranuclear cascade model is generally considered to be valid when the incident particle has a sufficiently small de Broglie wavelength to interact with individual nucleons. On this basis, a lower limit of $200 \mathrm{MeV}$ is usually quoted for the incident energy in nucleon-induced reactions. Here this statement is questioned. A pragmatic approach is used, which compares the predictions of the Liège intranuclear cascade model with available data at incident energy between 40 and $250 \mathrm{MeV}$. It is found that this model gives surprisingly good results at energies well below the limit mentioned above. Results are also compared with the predictions of other models commonly used in this energy range.
\end{abstract}

PACS. 25.40.-h Nucleon-induced reactions - 24.10.-i Nuclear-reaction models and methods - 24.10.Lx Monte Carlo simulations (including hadron and parton cascades and string breaking models)

\section{Introduction}

The intranuclear cascade (INC) model has been proven to be very successful in the description of the main features of nucleus-nucleus, proton-nucleus and antiproton-nucleus reactions in the $\mathrm{GeV}$ range $[1-3]$. In fact this model provides a good description of the first stage of the reaction process, characterized by hard nucleon-nucleon (or, more generally, baryon-baryon) scatterings, and predicts satisfactorily the high-energy part of particle spectra. In general, the INC model should be supplemented by an evaporation (after-burner) model to account for the later stage of the reaction, when softer processes take place, leading to the low-energy (evaporation-like) part of particle spectra.

The basic premise of the INC model considers the reaction process as a succession of binary collisions, well separated in time and space. This picture is generally justified by the mere comparison of the de Broglie wavelength $\lambda_{\mathrm{B}}$ with the average distance $d$ between nucleons, an argument first proposed by Serber some fifty years ago [4]. It is only recently that a sounder argument appeared, although some indication could already be found in works on Glauber's theory. Several authors have shown how to derive, starting from Schrödinger, Liouville or KadanoffBaym equations, a transport equation exhibiting a collision term [5-8]. The INC model does not solve this transport equation directly, but amounts to evaluating the colli-

\footnotetext{
a e-mail: cugnon@plasma.theo.phys.ulg.ac.be

b e-mail: P.Henrotte@ulg.ac.be
}

sion term by a stochastic method [9]. Whatever the starting point, all derivations rely on a basic assumption in order to arrive at the collision term: the scattering wave for the relative motion of two nucleons should be asymptotic before another collision involving one of these nucleons takes place. In other words, a collision should be over before the next one begins. Separation of collisions thus requires

$$
v t_{\text {coll }} \ll d, \lambda_{\mathrm{B}} \ll d,
$$

where $v$ is the average relative velocity and $t_{\text {coll }}$ is the time span during which the relative motion wave packet is not asymptotic, in some sense the collision time. Furthermore, the INC model relies on classical trajectories. Therefore, to be valid, $\lambda_{\mathrm{B}}$ should be much smaller than the range of interaction $r_{0}$. The quantity $t_{\text {coll }}$ is at least equal to $r_{0} / v$, the time of passage. Altogether, the presumed condition of validity of the INC model can be written as

$$
\lambda_{\mathrm{B}} \ll r_{0} \leq v t_{\mathrm{coll}} \leq d .
$$

On this basis, it has been considered for a long time that the INC model could not be valid for collisions induced by projectiles with a kinetic energy per nucleon of less than $250 \mathrm{MeV}$. Occasional applications of the INC model at lower energy produced however satisfactory agreement with experimental data. In particular, it has recently been shown that many properties of particle spectra in heavyion reactions at less than $100 \mathrm{MeV}$ incident kinetic energy per nucleon can result from the collision regime as embodied by INC models [10,11]. 
It is hard to establish the lower limit of validity by relying solely on the present theoretical knowledge. Condition (2) should be considered as a sufficient, but not a necessary condition. Furthermore, the quantity $v$ in eq. (2) does not only correspond to the initial conditions but also to the subsequent collisions.

In this paper, we will try to investigate the lower limit of validity of the INC model for nucleon-induced reactions on a purely empirical basis, just by comparing INC predictions with experimental data for various systems and various energies. More precisely, we want: i) to assess the validity of the INC model for decreasing incident energy; ii) to investigate the reasonable modifications of the model which are able to improve its predictive power at low energy. We will use the version of the Liège INC model described in sect. 2 . Whenever necessary, we will add the evaporation model of Dresner [12].

In sect. 2, we recall the most prominent features of our INC model. Section 3 is devoted to an extensive comparison with experimental data and to an evaluation of the predictive power of the model. Section 4 contains a discussion of the influence of the implementation of the Pauli blocking on the number of collisions in the INC model and on its predictions. It also compares our results with those of the alternative models used for nucleon-induced reactions in the energy range under interest. We give our conclusions in sect. 5, which also contains some remarks on the use of INC models at low energy in transport codes.

\section{The Liège INC model}

In this paper we consider the INC model as it is ordinarily used in transport calculations of particles in thick targets, a task which is of uttermost importance for the conception and design of accelerator-driven systems (ADS) and of modern powerful neutron spallation sources (see, for instance, ref. [13] for a review). In other words, we do not consider the INC model as predicting the total reaction cross-section (which in the above-mentioned calculations is taken from another model or from experiment [14]), but as a tool to describe the collision process in the proper impact parameter range.

We use the Liège INC model as cast in the numerical code INCL3. It differs from the version INCL2 described in ref. [2] by a single feature that is explained below. A complete description can be found in ref. [15] and in references cited therein. It is sufficient here to recall that the collision mechanism is assumed to proceed from a succession of binary collisions well separated in space and time. The fate of all particles is followed as time evolves. The particles travel along straight-line trajectories until two of them reach their minimum distance of approach, in which case they can be scattered provided the value of this distance is small enough, or until they hit the border of the potential well, supposed to describe the nuclear target mean field. Additional features are: 1) initial positions of target nucleons are taken at random in the spherical nuclear target volume with a sharp surface; 2) initial momenta are generated stochastically in a Fermi sphere; 3) relativistic kinematics is used; 4) isospin degrees of freedom are introduced for all types of particles and isospin symmetry is respected; 5) Pauli principle is enforced by means of statistical blocking factors; 6) spectator nucleons are not allowed to collide ${ }^{1}$.

The INCL3 version differs from the INCL2 one by a single feature: simulation runs are stopped when the excitation energy vanishes. Let us elaborate a bit on this point. For a reaction induced by a nucleon of incident kinetic energy $T_{\text {lab }}$, energy conservation implies

$$
T_{\mathrm{lab}}=K_{\mathrm{ej}}+W_{\pi}+E^{*}+S .
$$

In this equation, $K_{\mathrm{ej}}$ is the kinetic energy of the ejectiles, $W_{\pi}$ is the total energy of the produced pions, $E^{*}$ is the excitation energy of the remnant and $S$ is the separation energy, i.e. the minimum energy necessary to remove the ejected nucleons. Within the INC model, the quantities in eq. (3) can be evaluated at any time. Furthermore, ejectiles are considered as the particles located outside the potential well and $E^{*}$ can be expressed in terms of the kinetic energy of the particles in the remnant, as explained in ref. [2]. We just quote the result

$$
E^{*}=\sum_{j \in A_{\mathrm{rem}}} T_{j}-\sum_{j=1}^{A_{T}} T_{j}^{0}+\left(N_{\mathrm{ej}}-1\right) T_{\mathrm{F}} .
$$

In this relation $T_{j}$ is the actual kinetic energy of nucleon $j$ in the remnant, $T_{j}^{0}$ is the initial kinetic energy, $A_{\text {rem }}$ is the number of nucleons inside the target volume, $N_{\mathrm{ej}}$ is the number of ejected nucleons and $T_{\mathrm{F}}$ is the Fermi kinetic energy. Pauli blocking requires that particles cannot reach occupied states in the Fermi sea, guaranteeing that $E^{*}$ remains always positive. In our standard INC model, we use a stochastic implementation of the Pauli principle (see ref. [2]). Furthermore, the initial state is also generated stochastically, introducing fluctuations in the Fermi sphere occupancy. These features allow occasionally particles to overpopulate the Fermi sphere, which sometimes leads to negative $E^{*}$ (eq. (3)). In the INCL3 version, the latter unphysical effect is avoided by stopping a run when $E^{*}$, checked at any collision, becomes vanishingly small.

Here, we will also investigate the implementation of the strict Pauli blocking, which forbids collisions leading to nucleons with momentum smaller than the Fermi momentum $p_{\mathrm{F}}$. This prevents the Fermi sphere from being overpopulated. On the other hand, it does not accommodate the possible repopulation of the depleted parts of the Fermi sphere [2]. In the low-energy range, this depletion is quite small. We can anticipate on the discussion of sect. 4 and state that the results are generally better with the strict Pauli blocking.

The cascade stage develops until a time $t_{\text {stop }}$, determined according to a criterion described in ref. [2], unless

\footnotetext{
1 Nucleons are divided into participants and spectators. A participant is either the incident particle or any particle which has been hit by the latter or by another participant. The other particles are spectators.
} 
Table 1. Proton-induced and neutron-induced reactions, for which calculations have been made and compared with data from the indicated references. The numbers give the values of the total reaction cross-section (in mb) used in this work. The symbols indicate whether proton or neutron double differential cross-sections have been compared with experiment. A few references contain results for other isotopes than those which are mentioned.

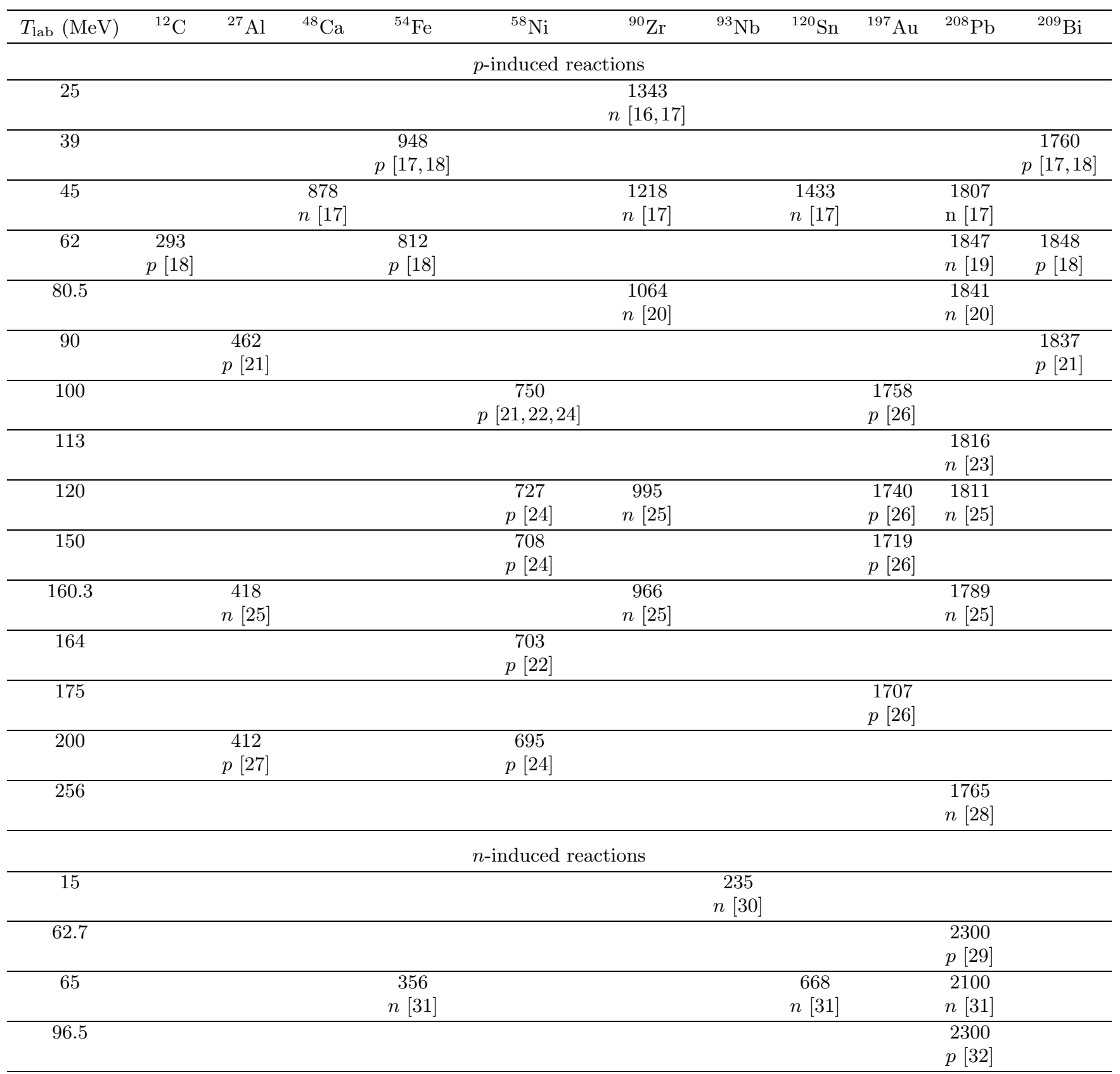

of course $E^{*}$ has vanished before. The necessary parameters $\left(E^{*}\right.$, charge and mass of the target remnant) are then transmitted to an evaporation module (here the Dresner code [12]), which simulates the subsequent de-excitation of the remnant.

\section{Comparison with experimental data}

\subsection{Introduction}

Table 1 contains a review of the most documented experimental data available for nucleon-induced reactions in the
25-200 MeV incident energy range. We did not include measurements that are too scarce: too few angles, limited range of emitted particle energy, etc. We have calculated double differential cross-sections for nucleon emission resulting from all reactions quoted in table 1. Only a few illustrative cases are shown below, the other ones giving similar results. For all results shown in this section, the strict Pauli blocking is used. Due to the often scattered experimental values of the total reaction cross-sections, we used, most of the time, the value given by the systematic of Wellish and Axen [33] (see sect. 5). For each case 


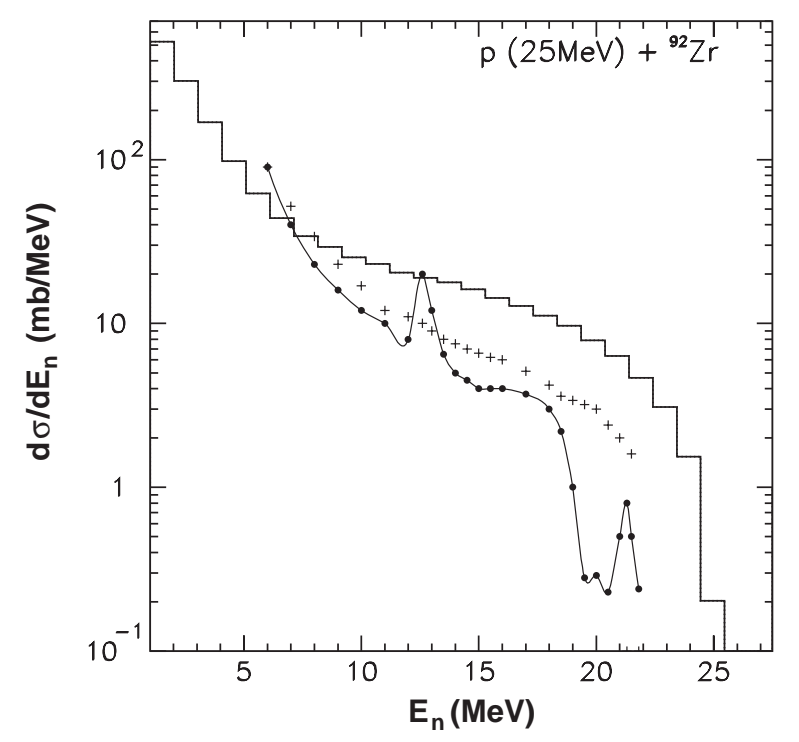

Fig. 1. Energy differential cross-section for neutron production in proton-induced reactions on ${ }^{92} \mathrm{Zr}$ at $25 \mathrm{MeV}$ incident energy. Data (dots) are taken from ref. [16] (experimental errors, not shown, are roughly 25\%) and are compared with the predictions of our model (histograms) and with those of GeometryDependent Hybrid (GDH) model (crosses), quoted in ref. [16].

shown below, more than 200000 runs have been accumulated. For double differential cross-sections, this means an average statistical uncertainty of about $7 \%$ on the average. Of course, the accuracy can be worse when the phase space is scarcely populated (see fig. 4 below for large angles).

\subsection{Proton-induced reactions}

We start with neutron emission. We display in fig. 1 the results of our calculation of the neutron spectrum for the lowest incident energy considered here, namely $25 \mathrm{MeV}$. As expected, the results are not very good, although the main feature, namely the presence of a "pre-equilibrium" component in addition to the evaporative component (here for neutron energy $E_{n}$ smaller than $\sim 7 \mathrm{MeV}$ ), is roughly reproduced. The presence of peaks in the high-energy part of the experimental neutron spectrum, which correspond to coherent scattering (to low-lying states and to the isobaric analog resonance), is of course not reproduced by our model, which incorporates only incoherent processes. The end point of the neutron spectrum is also misplaced, because the $Q$-value for a $(p, x n)$ reaction vanishes in our model, whereas the experimental value is equal to $-1.8 \mathrm{MeV}$ for a ${ }^{92} \mathrm{Zr}$ target. In addition, the magnitude of the cross-section is overestimated at large neutron energy.

Figure 2 shows our results for the same quantity along with experimental data, concerning proton-induced reactions on three targets at $45 \mathrm{MeV}$. It can be seen that the results of the model compare reasonably well with the data, except for the lightest target, where the cross-section is underestimated, especially at large neutron energy. We

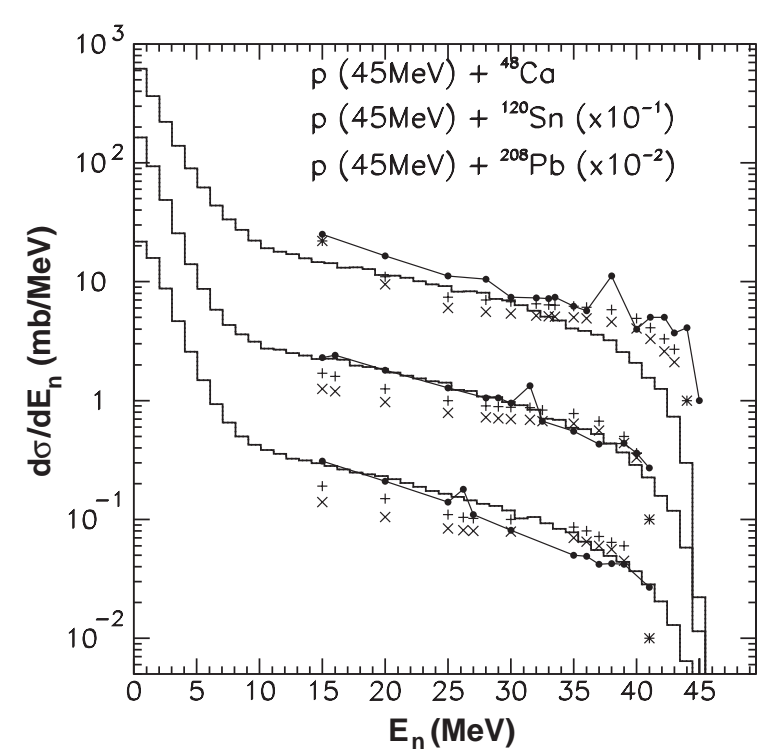

Fig. 2. Energy differential cross-section for neutron production in proton-induced reactions on three targets at $45 \mathrm{MeV}$ incident energy. Data (dots, with an accuracy of about 20\%) are taken from ref. [17] and are compared with the predictions of our model (histograms) and with those of the GDH model, the quantity $\lambda_{+}$(see eq. (5)) being defined in terms of the nucleon-nucleon cross-section $(x)$ or in terms of the imaginary part of the optical-model potential $(+)$. See the text for details.

disregard of course coherent scattering, whose importance anyway decreases rapidly with increasing incident energy. The top panel of fig. 3 shows that the same kind of agreement is achieved at $80.5 \mathrm{MeV}$ on a $\mathrm{Pb}$ target.

A more detailed comparison is provided by the bottom panel of fig. 3, which shows the predicted neutron double differential cross-section for the $p(80.5 \mathrm{MeV})+\mathrm{Pb}$ system. As a rule in this article and for the sake of clarity, double differential cross-sections are displayed after multiplication by $10^{0}, 10^{-1}, 10^{-2}$, etc., for successive angles in increasing order. One can see from fig. 3 that the agreement is rather good in the whole range of neutron energy above $20 \mathrm{MeV}$, i.e. the pre-equilibrium component, except for the smallest and the largest angle, for which the crosssection is slightly underestimated. We have to mention that the authors of ref. [20] acknowledged that their measurements between $82^{\circ}$ and $106^{\circ}$ are underestimated by a factor lying between 1.5 and 2 (a conclusion derived from continuity arguments). They nevertheless published their uncorrected data, which we reproduced here (this is also true for figs. 4 and 5). As a consequence, the agreement between our calculations and the experimental data is actually slightly less good than shown in fig. 3 for $95^{\circ}$ and $106^{\circ}$.

A similar comparison performed at higher energy is displayed in fig. 4 for a Zr target. Similar remarks are in order. The cross-section is nicely reproduced except at the smallest and the largest angles, for which the cross-section is underpredicted. Note that the smallest angle in this case is smaller than in the previous case and for such a small angle, the shape of the neutron spectrum is not well reproduced. Note also that the experimental curve at $95^{\circ}$ 

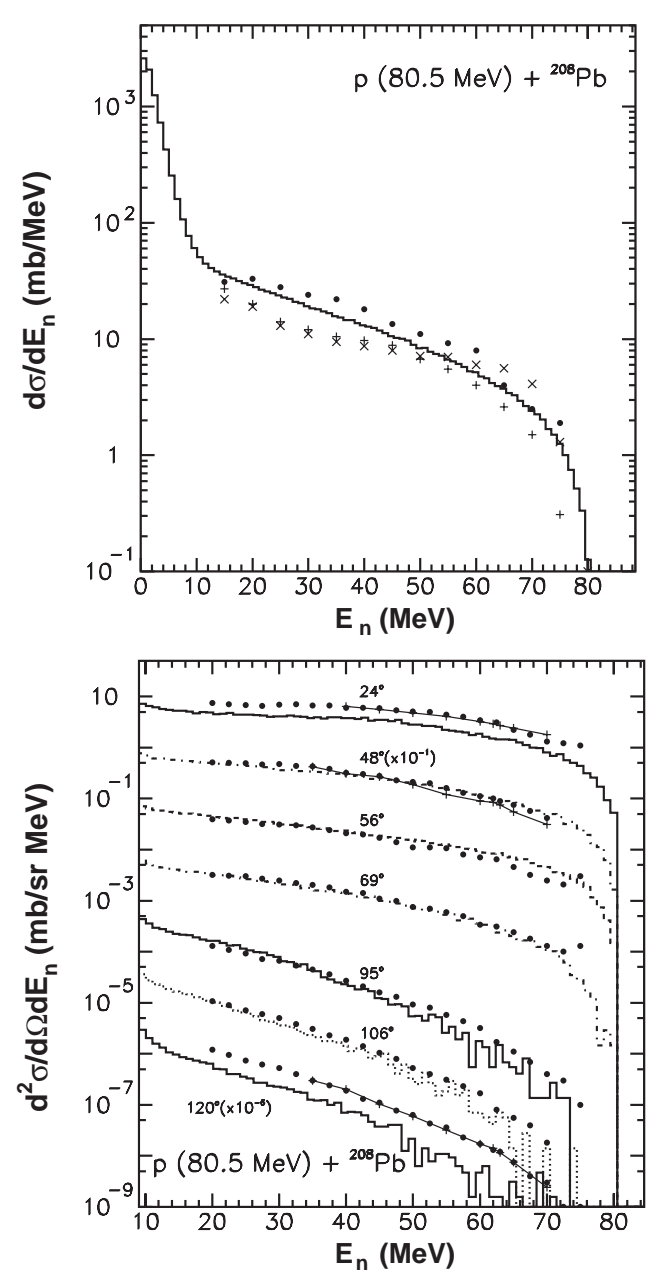

Fig. 3. Energy differential (top panel) and double differential (bottom panel) neutron cross-section for proton-induced reactions on ${ }^{208} \mathrm{~Pb}$ at $80.5 \mathrm{MeV}$. Data (dots, with an uncertainty of about 20\%) are taken from ref. [20]. On the bottom panel, the end of the experimental spectra (above $70 \mathrm{MeV}$ ) containing the pure elastic peak and the coherent excitation peaks to some low-lying states, is not shown. For the sake of clarity, the double differential cross-sections are displayed after multiplication by $10^{0}, 10^{-1}, 10^{-2}$, etc., for angles in increasing order (same convention for the following figures). On the top panel, data are compared with the predictions of our model (histograms) and with those of the Hybrid $(\mathrm{H})$ and Geometry-Dependent Hybrid (GDH), as quoted in ref. [20]. These are indicated by the + and the $\times$ symbols, respectively. On the bottom panel, data are compared with the predictions of our model (histograms) and with the ones of the Multi-Step Direct (MSD) model of ref. [48] (continuous lines, as quoted in ref. [20]). The experimental data at $95^{\circ}$ and $106^{\circ}$ are underestimated by a factor between 1.5 and 2 .

should be increased by a factor $\sim 1.5$, which would improve the agreement shown in fig. 4. Going still higher in energy, we report in fig. 5 on our calculations at $160 \mathrm{MeV}$. We prefer to display the results for the angular distributions, which are usually considered as a more sensitive test than the shapes of the particle spectra. Although indeed the agreement between our predictions and the data is not as good as in the two previous figures, the main features

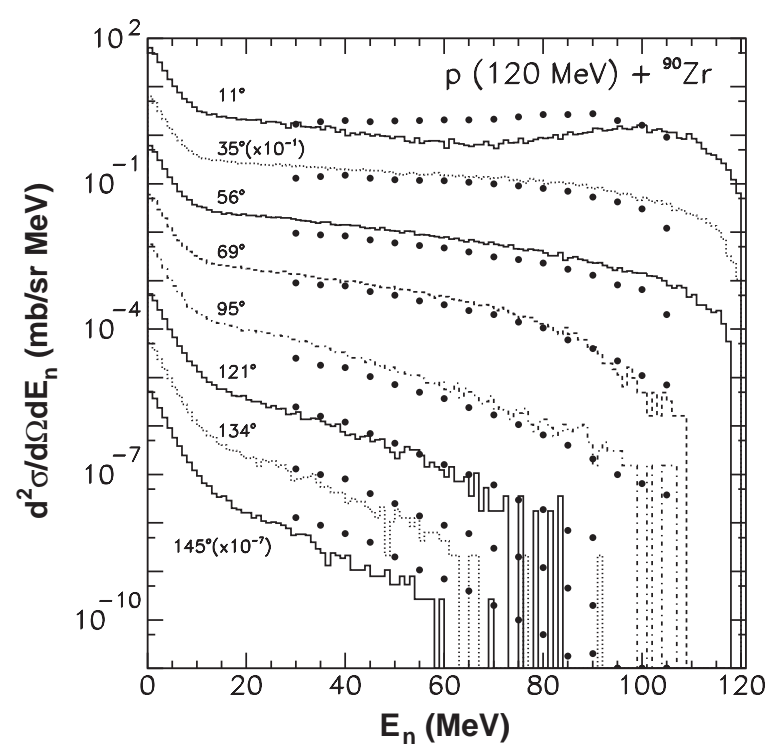

Fig. 4. Double differential neutron cross-section for protoninduced reactions on ${ }^{90} \mathrm{Zr}$ at $120 \mathrm{MeV}$. Data (dots, experimental uncertainty of about 20\%) are taken from ref. [25] and are compared with the predictions of our model (histograms). The experimental data at $95^{\circ}$ are underestimated by a factor $\sim 1.5$.

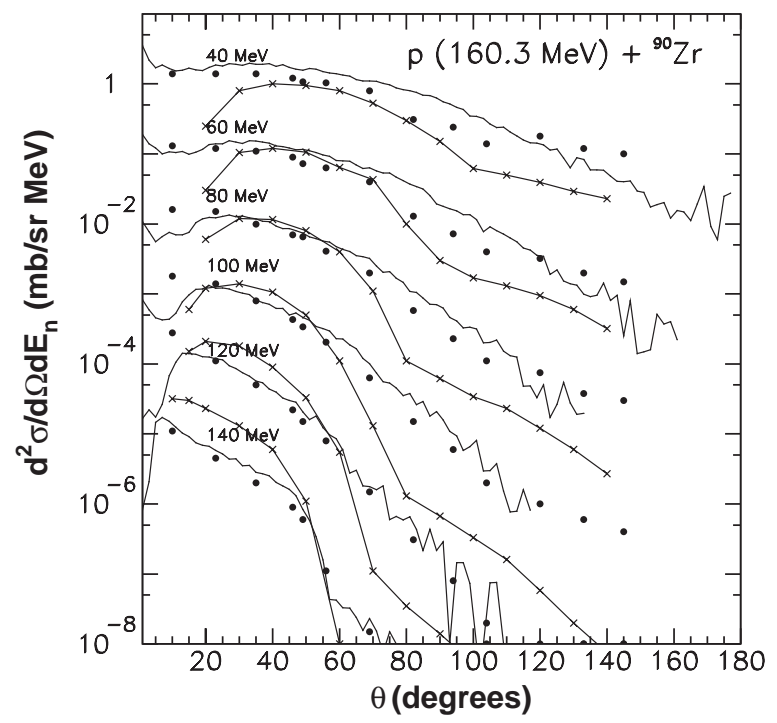

Fig. 5. Double differential neutron cross-section for protoninduced reactions on ${ }^{90} \mathrm{Zr}$ at $160.3 \mathrm{MeV}$, plotted versus the neutron emission angle. Data (dots, experimental uncertainty of about 20\%) are taken from ref. [25] and are compared with the predictions of our model (lines) and with those of the GDH model (lines with crosses, as quoted in ref. [25]). The experimental data between $82^{\circ}$ and $102^{\circ}$ are marred by a systematic error. They should be multiplied by a factor between 1.5 at small energies and unity at large energies.

of the angular distributions are satisfactorily reproduced except at small angles $\left(\sim 10^{\circ}\right)$ for neutron energy above $\sim 60 \mathrm{MeV}$ and, to a smaller extent, at large angles (above $120^{\circ}$ ) for neutron energy below $\sim 80 \mathrm{MeV}$. Finally, for the sake of comparison, we give in fig. 6 the results obtained at $256 \mathrm{MeV}$, close to the commonly accepted lowest energy for the validity of the INC model. In conclusion, 


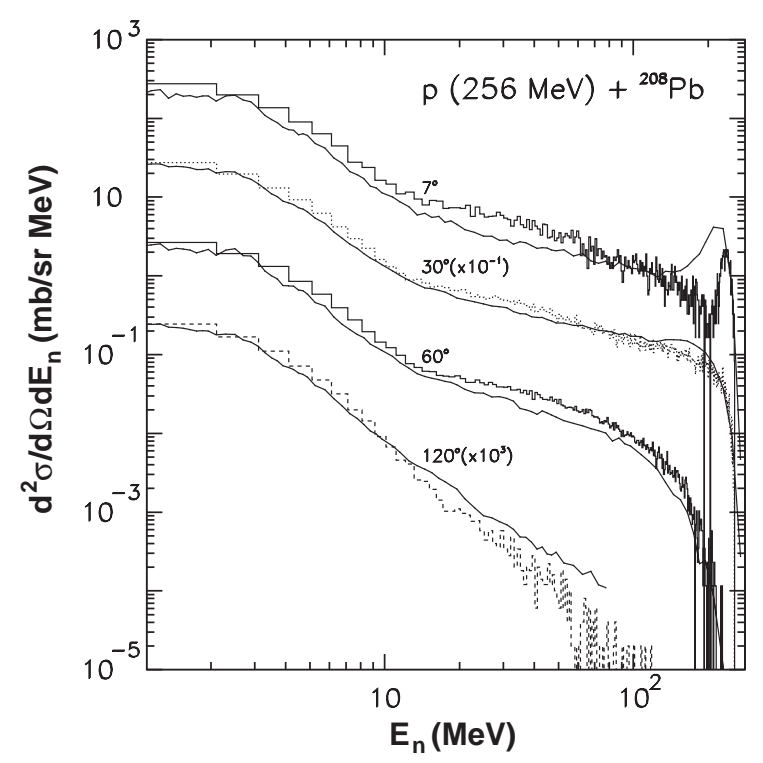

Fig. 6. Double differential neutron cross-section for protoninduced reactions on ${ }^{208} \mathrm{~Pb}$ at $256 \mathrm{MeV}$. Data (lines) are taken from ref. [28] and are compared with the predictions of our model (histograms). The experimental uncertainties are of the order of $10 \%$.

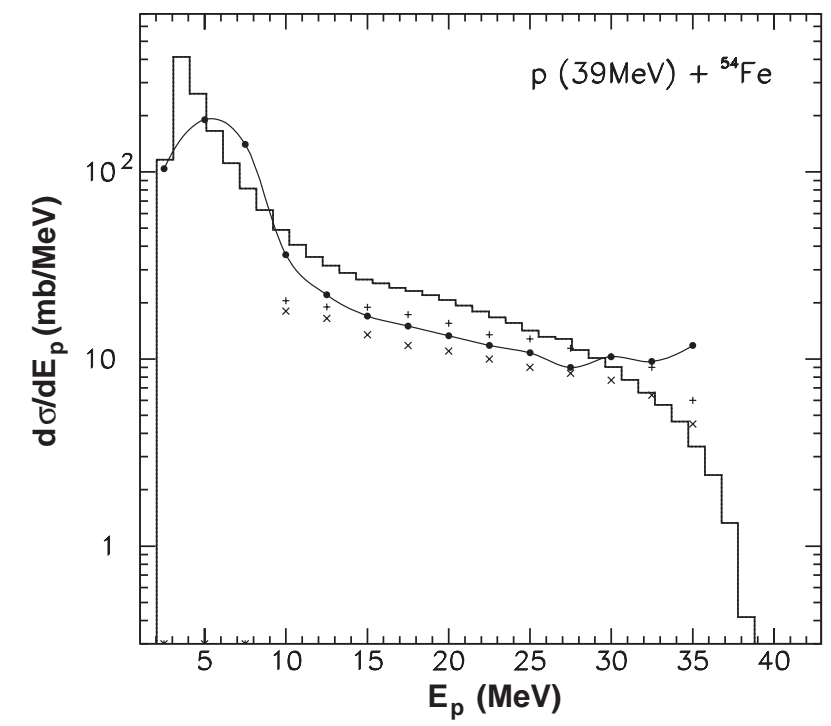

Fig. 7. Energy differential cross-section for proton emission in proton-induced reactions on ${ }^{54} \mathrm{Fe}$ at $39 \mathrm{MeV}$. Data (dots, experimental uncertainties of $\sim 20 \%$ ) are taken from ref. [18] and are compared with the predictions of our model (histograms) and with the predictions of the GDH model, as quoted in ref. [17], using a mean free path related to the imaginary part of the optical model $(+)$ or to the nucleon-nucleon cross-section $(\times)$.

one can see that the degree of agreement met by our model is smoothly deteriorating as the incident energy is decreasing, but is largely acceptable at much lower energy than $256 \mathrm{MeV}$. We will come back to this point.

We now turn to proton emission. We start at low energy. Our results for the energy differential cross-section

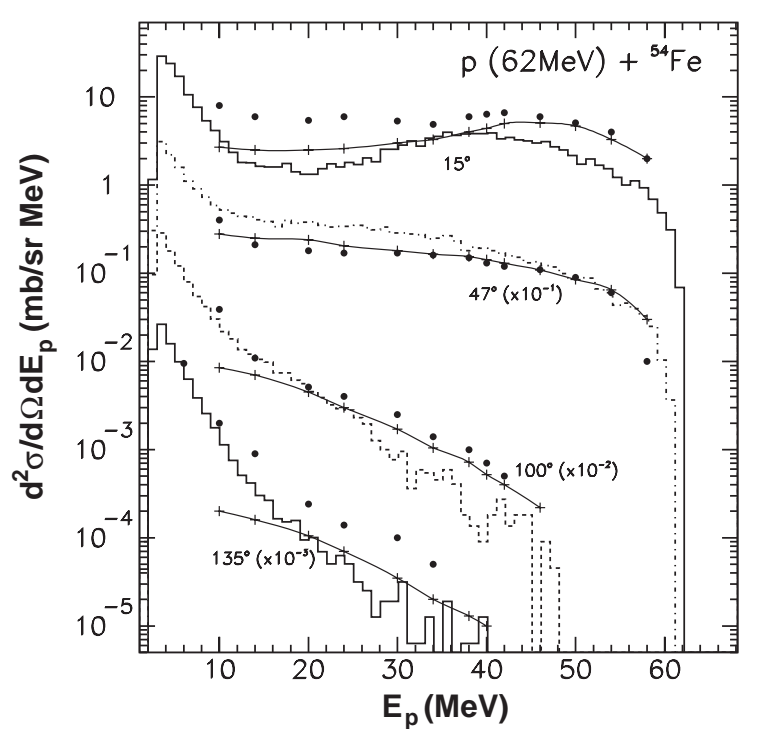

Fig. 8. Double differential proton cross-section for protoninduced reactions on ${ }^{54} \mathrm{Fe}$ at $62 \mathrm{MeV}$. Data (dots, experimental uncertainties of $\sim 10 \%$ ) are taken from ref. [18] and are compared with the predictions of our model (histograms) and with those of ref. [49] (lines with crosses).

for the $(p, x p)$ reaction on a ${ }^{54} \mathrm{Fe}$ target at $39 \mathrm{MeV}$ are displayed in fig. 7, along with the experimental data of ref. [17]. One can see that the agreement is not really good, especially at high outgoing proton energy. This is a constant feature of the $(p, x p)$ results: the coherent scattering leading to low-lying target states is more intense than the coherent scattering in $(p, x n)$ reactions at the same incident energy. Note that this coherent scattering occurs at small emission angle only. Interestingly, the measurements of ref. [17] extend to the evaporation domain. It can be seen that our model gives reasonable, though not really good, results for this part.

Figure 8 shows how the situation evolves when going to a somewhat higher energy, for the double differential proton cross-section this time. The agreement is not very good, and should be contrasted with the one obtained in figs. 2-3. In this energy range, our results turn out to be noticeably less good for $(p, x p)$ reactions than for $(p, x n)$ reactions. The comparison is getting better when the incident energy is increased up to $90 \mathrm{MeV}$ (see fig. 9). Disregarding the experimental peaks at large energy, which arise from coherent scattering, the overall agreement is rather satisfactory. However, one can detect a systematic discrepancy: the experimental data are overestimated by our model at the largest angles quoted in the figure and underestimated at small angles. As a consequence, a good agreement is obtained for some intermediate angles, which turn out to be around $30^{\circ}$ for the two targets considered in fig. 9.

In fig. 10, we move to higher incident energies. Here one sees that the agreement has very much improved. There are some tendencies: i) the agreement is increasing with incident energy, being excellent at $200 \mathrm{MeV}$; ii) the performances of the model are rather bad for the smallest 

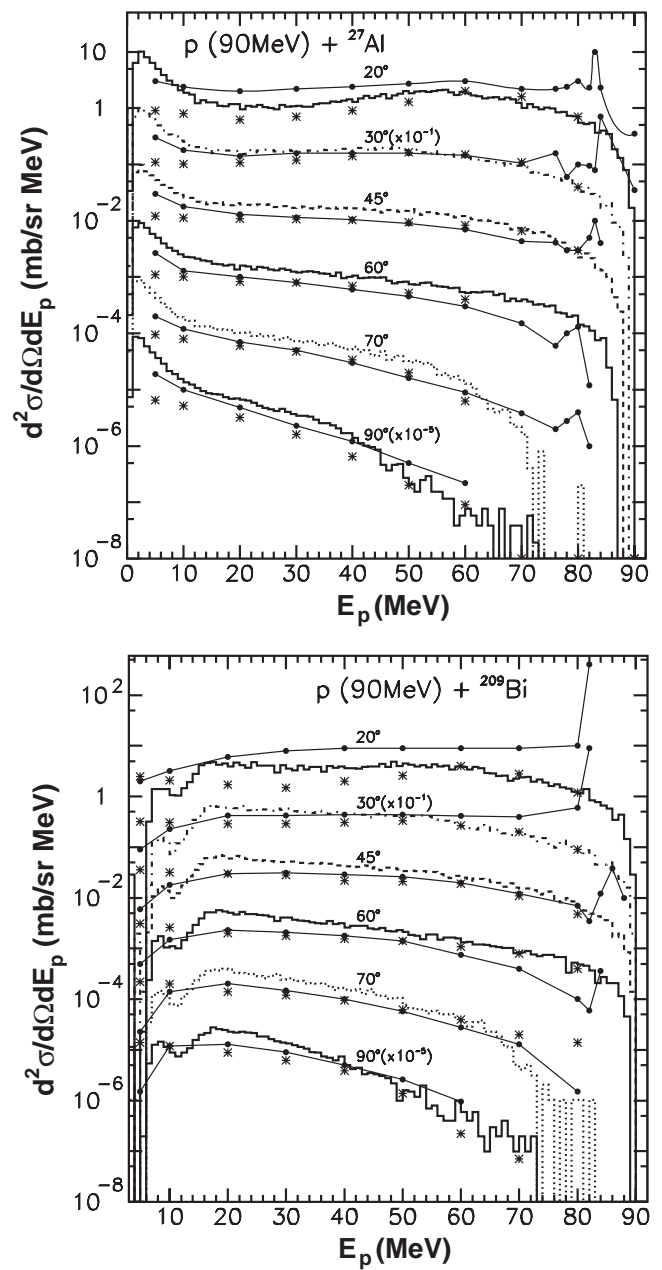

Fig. 9. Double differential proton cross-section for protoninduced reactions on ${ }^{27} \mathrm{Al}$ (top panel) and on ${ }^{209} \mathrm{Bi}$ (bottom panel), at $90 \mathrm{MeV}$. Data (dots) are taken from ref. [21] and are compared with the predictions of our model (histograms) and those of ref. [34] (crosses). The experimental uncertainty is of the order of $10 \%$.

angle $\left(15^{\circ}\right)$; iii) at this angle, the cross-section is substantially underestimated for energies of the emitted protons lying around half the incident energy; iv) there is also a slight overestimation for $50^{\circ}$ and $70^{\circ}$ when the energy of the emitted protons is just above the evaporation range. We have very similar results for the Au target at the same incident energies.

\subsection{Neutron-induced reactions}

Extensive data are even more scarce than for protoninduced reactions. In fig. 11, results for the $n+{ }^{93} \mathrm{Nb}$ system at $15 \mathrm{MeV}$ incident energy are displayed, along with the data. The agreement is surprisingly good, which is perhaps accidental. We have no justification for it, at least. More extensive and reliable data have been obtained recently at $62.7 \mathrm{MeV}$ [29]: they refer to proton production and are displayed in fig. 12. Globally, the agreement with data is similar to, but slightly less good than the one prevailing in proton-induced reactions at the same inci-
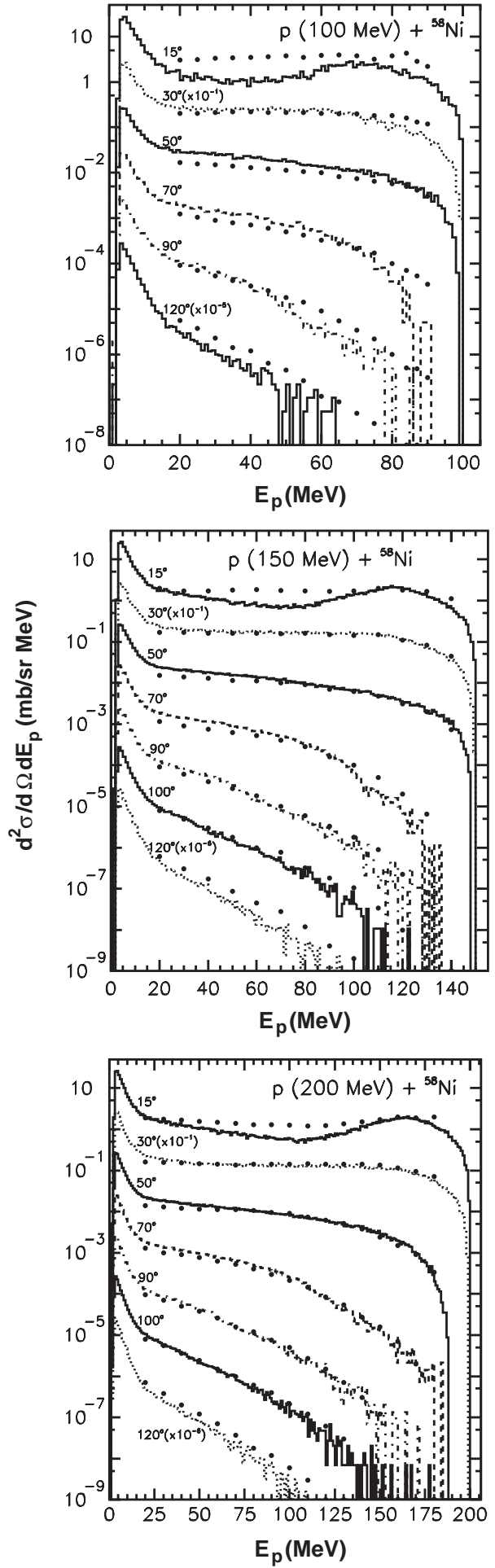

Fig. 10. Double differential proton cross-section for protoninduced reactions on ${ }^{58} \mathrm{Ni}$ at 100,150 and $200 \mathrm{MeV}$. Data (dots) are taken from ref. [24]. They are compared with the predictions of our model (histograms). The experimental uncertainties are lying between 10 and $20 \%$.

dent energy (see figs. 2-3). If one looks a little bit more into the details, one can observe that the predictions are slightly better for the most forward angle (here $20^{\circ}$ ) and slightly worse for the largest angle. For all angles there 


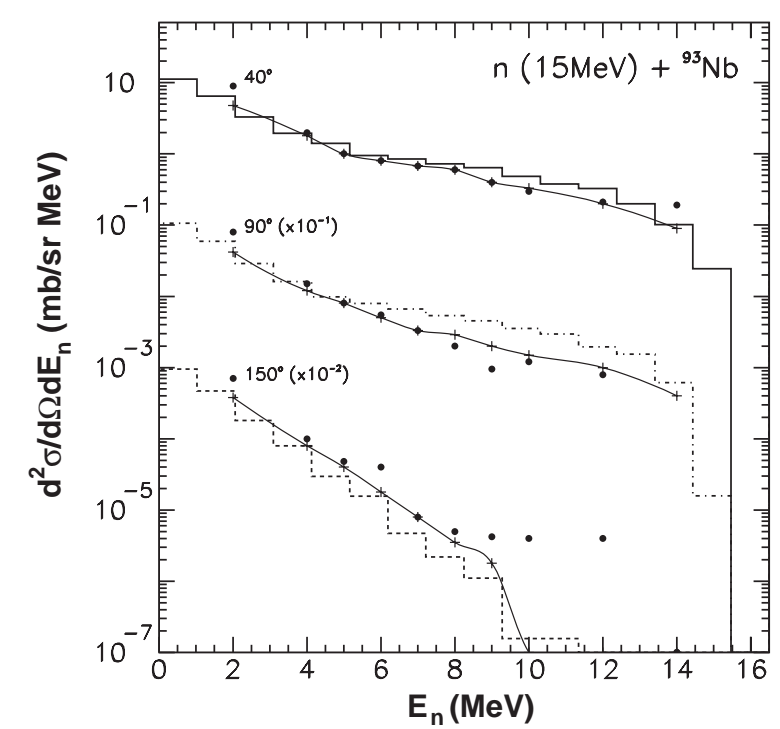

Fig. 11. Double differential neutron cross-section for neutroninduced reactions on ${ }^{93} \mathrm{Nb}$ at $15 \mathrm{MeV}$. Data (dots) are taken from ref. [30]. They are compared with the predictions of our model (histograms) and those of ref. [34] (lines with crosses). The experimental uncertainties are of the order of $20 \%$.

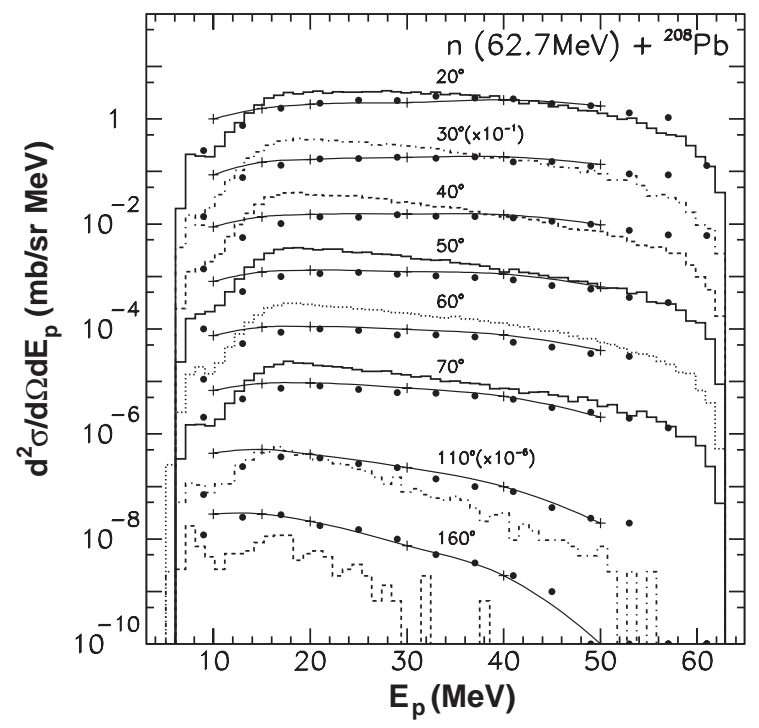

Fig. 12. Double differential proton cross-section for neutroninduced reactions on ${ }^{208} \mathrm{~Pb}$ at $62.7 \mathrm{MeV}$. Data (dots, experimental uncertainty of about 10\%) are taken from ref. [29]. They are compared with the predictions of our model (histograms) and those of the TALYS code system [35] (continuous lines with crosses, taken from ref. [36]).

is a systematic overestimation of the cross-section around 15-20 MeV. This feature seems to come from a too high Coulomb barrier in the cascade, which is accompanied by a probably too low barrier in the Dresner evaporation model (responsible for the small peaks around $6 \mathrm{MeV}$ in some of the proton spectra). The lack of data for low-energy produced particles did not allow us to investigate a possible removal of this deficiency. In fig. 13, we investigate the $(n, x n)$ reaction on a ${ }^{54} \mathrm{Fe}$ target at about the same inci-

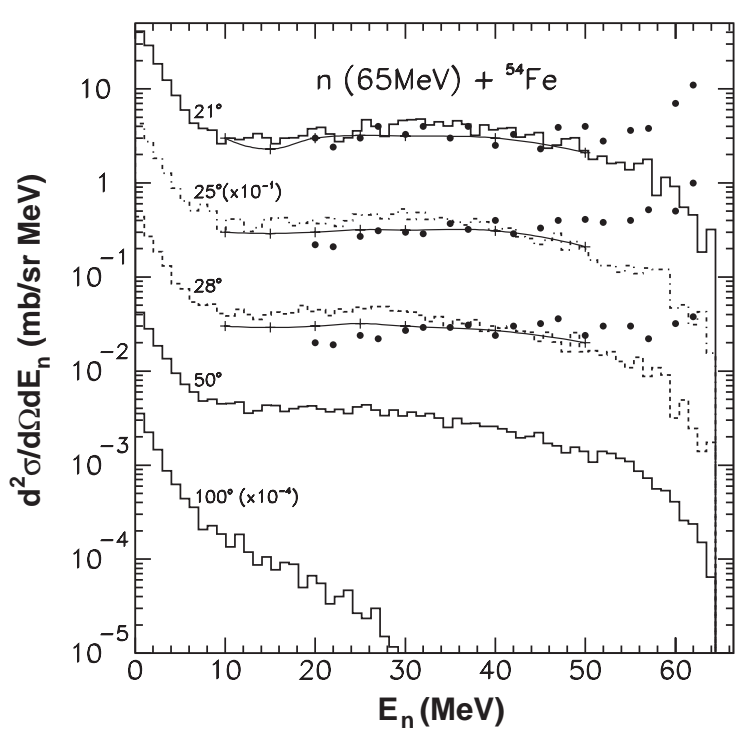

Fig. 13. Double differential neutron cross-section for neutroninduced reactions on ${ }^{54} \mathrm{Fe}$ at $65 \mathrm{MeV}$. Data (dots) are taken from ref. [31]. They are compared with the predictions of our model (histograms) and with those of the model from ref. [50] (lines with crosses, as quoted in ref. [31]). The experimental uncertainties are about $25 \%$ for $21^{\circ}$ and $15 \%$ for the other angles.

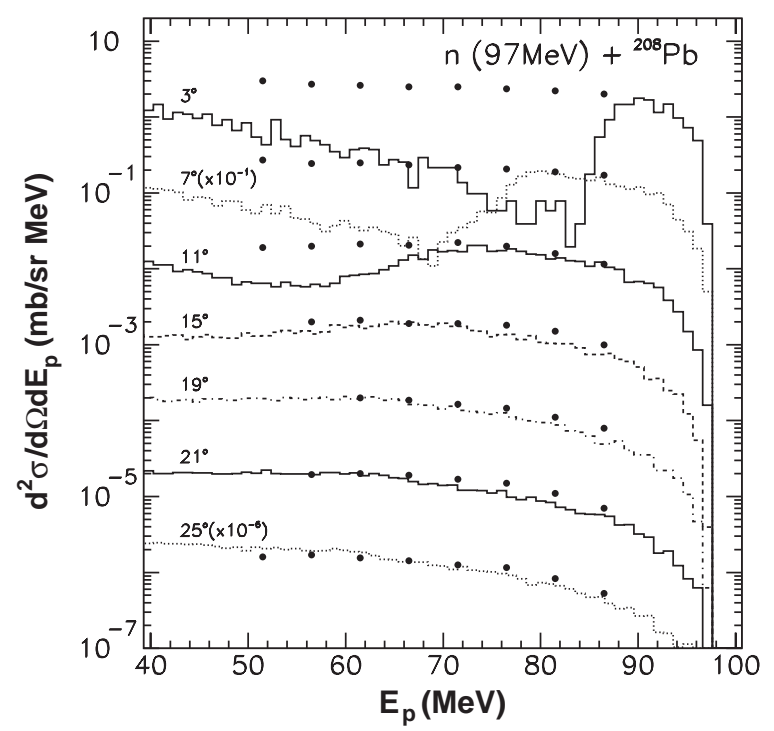

Fig. 14. Double differential proton cross-section for neutroninduced reactions on ${ }^{208} \mathrm{~Pb}$ at $97 \mathrm{MeV}$. Data (dots), from ref. [32], are compared with the predictions of our model (histograms). The experimental uncertainties are about $4-5 \%$.

dent energy. The overall agreement is very similar, except, of course, for the experimental rise at large detected neutron energy, which comes from coherent processes, as we already mentioned for other systems.

Finally, we show in fig. 14 the comparison between our predictions and the data for a higher incident energy, namely $97 \mathrm{MeV}$. For angles above $15^{\circ}$, the agreement is rather good and similar to the one observed for proton-induced reactions (see figs. 4,9-10). The emission at $11^{\circ}$ is slightly underpredicted around $60 \mathrm{MeV}$, like 
in proton-induced reactions (see fig. 4). Interestingly, in this case, one has measurements for very small angles, for which our model largely underpredicts the yields around $80 \mathrm{MeV}$. We will come back to this point.

\subsection{Summary}

We have shown in many cases that the INC model yields surprisingly good results well below the often quoted lowest incident energy where it could be valid. The agreement looks sometimes a little bit erratic (compare, for instance, the bottom panels of figs. 3 and 9 ), pointing to a possible dependence of the data on the detail of the structure of the target nuclei (of course, an erratic dependence upon the target mass or upon the energy cannot come from our model where both the description of the initial target state and the elementary cross-sections are smooth functions). But, these "accidents" are small fluctuations on a smooth variation. Trying to summarize the general trend, one can say our model gives good results for $(p, x n)$ reactions above $45 \mathrm{MeV}$. The agreement improves with increasing incident energy and with increasing target mass. It is also generally better for intermediate angles than for forward and backward angles. For $(p, x p)$ reactions, the trends are the same, but a little bit more pronounced: when varying the parameters mentioned above, the agreement worsens faster than in the $(p, x n)$ case. For neutron-induced reactions, the number of cases investigated is much lower and does not really entitle to draw precise conclusions, but presumably the same trends are present, with a systematically slightly less good agreement.

\section{Discussion}

\subsection{Analysis of our INC results}

We will mainly discuss two features: the effect of the Pauli blocking and the number of collisions. As we already mentioned, we presented results with a "strict" Pauli blocking, allowing only collisions when the momenta of the two outgoing nucleons are larger than the Fermi momentum $p_{\mathrm{F}}$. In the INCL3 model, originally designed for higher energies, a statistical Pauli blocking is implemented: when two nucleons $i$ and $j$ are going to suffer a collision at positions $\mathbf{r}_{i(j)}$ leading to a final state with momenta $\mathbf{p}_{i(j)}$, the phasespace occupation probabilities $f_{i}$ are evaluated by counting nearby nucleons in a small phase-space volume centered on the point $\left(\mathbf{r}_{i(j)}, \mathbf{p}_{i(j)}\right)$ and the collision is allowed or forbidden following the result of the comparison of a random number with the product $\left(1-f_{i}\right)\left(1-f_{j}\right)$. A comparison between the results using the two implementations of the Pauli blocking on a typical case is provided by figs. 3 and 15. There are two main effects on the cascade component (above $\sim 20 \mathrm{MeV}$ ) of the neutron spectra. First, the statistical Pauli blocking produces an enhancement of the cross-section at the high-energy edge of the spectra, especially at forward angles (less than $\sim 60^{\circ}$ ). Second, it generates a general increase at large angles (here larger than

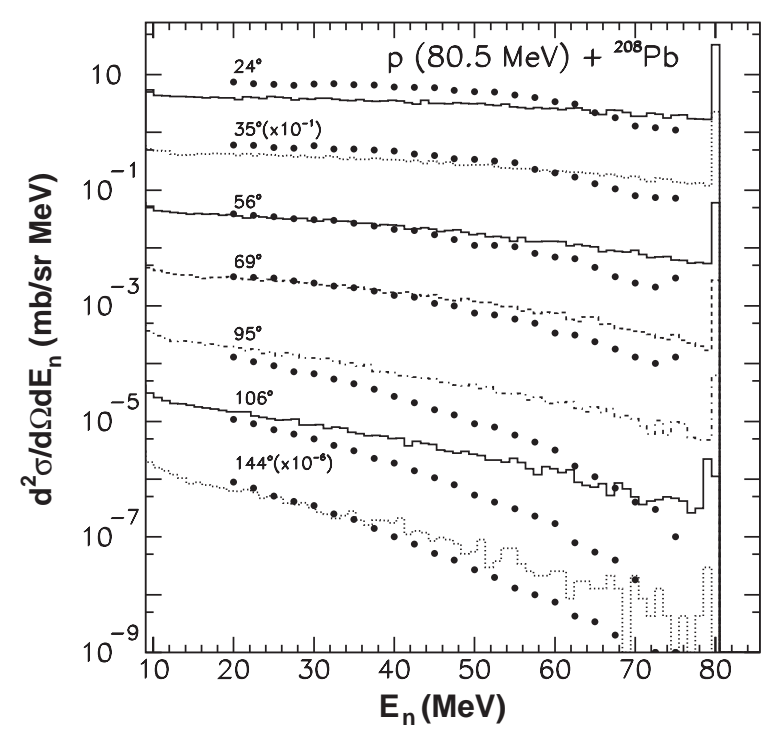

Fig. 15. Double differential neutron cross-section for protoninduced reactions on ${ }^{208} \mathrm{~Pb}$ at $80.5 \mathrm{MeV}$. Data (dots) are taken from ref. [20]. They are compared with the predictions of our model (histograms) using the statistical implementation of the Pauli blocking. This should be compared with fig. 3 (bottom panel), which displays the results for the strict Pauli blocking. See the text for details.

$\sim 90^{\circ}$ ). The first effect is easily attributed to the fluctuations in the filling of the initial Fermi sphere, which leave some free space in the phase space volume. As a result of the statistical implementation of the Pauli blocking, collisions are then able to fill these unphysical holes in the Fermi sea. Although the global effect on the neutron multiplicity is small (see table 2), it nevertheless produces a systematic distortion of the spectra in the so-called quasifree region. That is why we presented results obtained with the strict Pauli blocking, which in this region is undoubtedly better. The second effect of the statistical Pauli blocking is the increase of the cross-section at backward angles, in particular for particles ejected with high energy. This effect is less easy to understand, as (high-energy) backward neutrons are expectedly produced after a few hard collisions, not so sensitive to Pauli blocking.

We observed that the strict Pauli blocking is globally better than the statistical one for the double differential cross-section in all cases studied here. The difference between the results obtained with the different implementations is almost vanishing at $150-200 \mathrm{MeV}$ and increases when the incident energy decreases, as expected. In fact, we found that even better results are obtained with a "mixed" implementation, in which we impose the strict Pauli blocking for the first collision and the statistical implementation of the Pauli blocking for the remaining part of the events. With this choice, the spurious peak close to the incident energy at small angles is largely erased (as for the strict Pauli blocking) and the cross-section at large angles is enhanced (as in the statistical implementation). We do not discuss this point further, leaving it for a future work, but it seems to emphasize the necessity of taking 
Table 2. Comparison of several quantities calculated, for the $p+{ }^{208} \mathrm{~Pb}$ case (at 80.5 and $256 \mathrm{MeV}$ ), with three different implementations of the Pauli blocking. The mixed one corresponds to a strict application until the first collision is over, after which the statistical implementation is adopted. The quantities are the frequencies of absorption events (no ejected particle at the end of the cascade) and of transparent events (no collision), in percent, the average numbers of ejected neutrons and protons, the average excitation energy (in $\mathrm{MeV}$ ), the average number of collisions $\left(N_{\text {coll }}\right)$, the average number of blocked collisions $\left(N_{\mathrm{bl}}\right)$ and the average number of blocked collisions in which the incident nucleon is involved $\left(N_{\mathrm{bl}}^{1}\right)$.

\begin{tabular}{|c|c|c|c|}
\hline & \multicolumn{3}{|c|}{ Pauli blocking } \\
\hline & Statistical & Mixed & Strict \\
\hline \multicolumn{4}{|c|}{$p(80.5 \mathrm{MeV})+{ }^{208} \mathrm{~Pb}$} \\
\hline Absorption events (\%) & 5.18 & 5.18 & 6.77 \\
\hline Transparent events (\%) & 10.46 & 17.19 & 17.20 \\
\hline$\left\langle n_{\mathrm{ej}}\right\rangle$ & 0.79 & 0.82 & 0.73 \\
\hline$\left\langle p_{\mathrm{ej}}\right\rangle$ & 0.51 & 0.54 & 0.58 \\
\hline$\left\langle E^{*}\right\rangle$ & 32.9 & 36.7 & 41.9 \\
\hline$\left\langle N_{\text {coll }}\right\rangle$ & 5.03 & 5.06 & 2.24 \\
\hline$\left\langle N_{\mathrm{bl}}\right\rangle$ & 3.71 & 4.52 & 6.85 \\
\hline$\left\langle N_{\mathrm{bl}}^{1}\right\rangle$ & 1.51 & 2.09 & 3.03 \\
\hline \multicolumn{4}{|c|}{$p(256 \mathrm{MeV})+{ }^{208} \mathrm{~Pb}$} \\
\hline$\left\langle n_{\mathrm{ej}}\right\rangle$ & 1.54 & 1.57 & 1.50 \\
\hline$\left\langle p_{\text {ej }}\right\rangle$ & 1.07 & 1.11 & 1.17 \\
\hline$\left\langle N_{\text {coll }}\right\rangle$ & 8.41 & 8.47 & 4.77 \\
\hline$\left\langle N_{\mathrm{bl}}\right\rangle$ & 4.76 & 5.06 & 8.37 \\
\hline
\end{tabular}

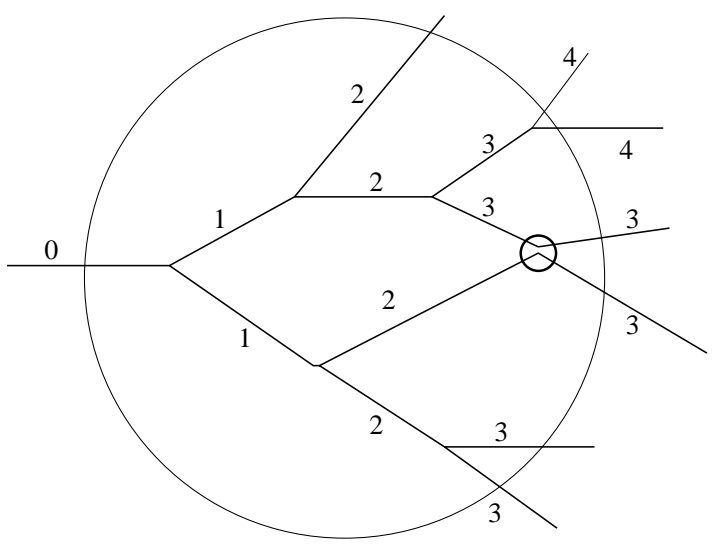

Fig. 16. Schematic representation of the arborescent structure of the cascade process. The values of the activation numbers for the participant nucleons are indicated. The small circle indicates the collision between two participant nucleons. See the text for details.

account of the possible repopulation of the depleted Fermi sea, even when, as here, this depletion is small.

It is interesting to analyze the results of our calculations in terms of $n_{\mathrm{a}}$, which we call the activation number and which indicates whether a nucleon participating in the cascade process has been activated early or late in the

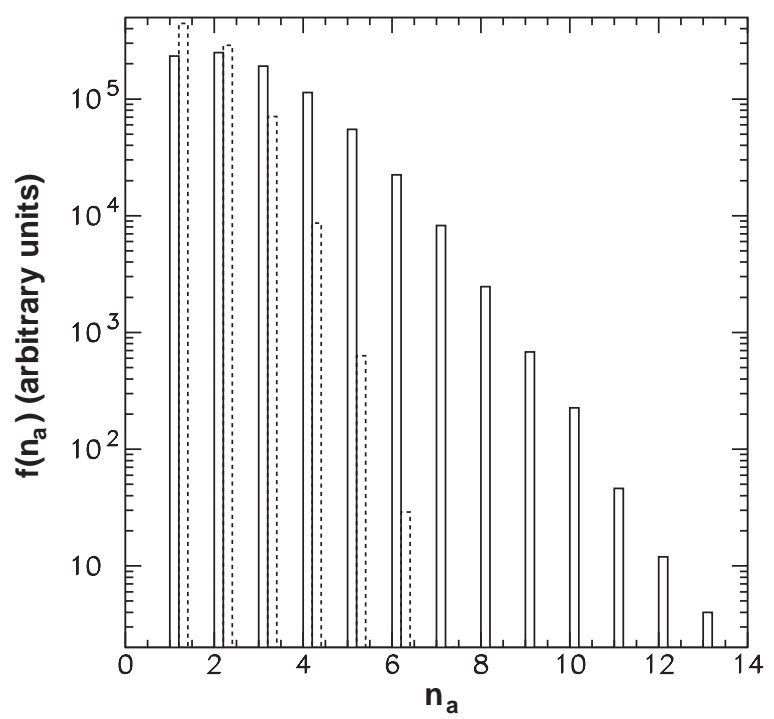

Fig. 17. Distribution of the activation numbers of the emitted particles, for the strict implementation of the Pauli blocking (dotted histogram) and for the statistical one (full histogram). The system under consideration is $p(80.5 \mathrm{MeV})+{ }^{208} \mathrm{~Pb}$. The distributions are given in arbitrary values, but for exactly the same number of events: the integrated distributions are thus proportional to the average nucleon multiplicities. See the text for details.

arborescent structure of the collision process (see fig. 16). At the beginning, $n_{\mathrm{a}}=0$ for all nucleons. After the first collision, $n_{\mathrm{a}}$ is put equal to unity for the two nucleons involved. Later on, in a collision between a participant $\left(n_{\mathrm{a}} \neq 0\right)$ and a spectator $\left(n_{\mathrm{a}}=0\right)$, the activation number of the participant is increased by one unit and the one of the former spectator is given the same value. It is easy to see from fig. 16 that $n_{\mathrm{a}}$ labels the bifurcations of the graph representing the successive collisions. It may happen, however, that branches of the graph coalesce, which occurs through collisions between two participants. After such a collision, the lowest activation number is increased by one unit and the same resultant value is set for the other one. Let us add that the activation number is not modified by the reflection of the nucleons on the nuclear surface. We finally recall that spectator nucleons are not allowed to collide with each other in the INCL3 model.

The average distribution of the activation number of the emitted particles (in the cascade stage) is given in fig. 17, both for the statistical and the strict Pauli blockings. The average value of $n_{\mathrm{a}}$ is smaller for the strict Pauli blocking, which expectedly suppresses more collisions than the statistical Pauli blocking. Similarly, the number of transparent events is indeed more important with the strict Pauli blocking ${ }^{2}$ (see table 2). Nevertheless, the average number of emitted nucleons remains the same, but the one of emitted neutrons (protons) slightly decreases (increases). The first result indicates that, very likely, the strict Pauli blocking essentially removes rather

2 These events are not taken into account for the evaluation of the particle emission cross-sections. 
soft collisions involving nucleons with small energy. The hard collisions, presumably more efficient to eject nucleons, are essentially not more suppressed by the strict Pauli blocking than by the statistical one. The second result needs a more subtle explanation. The number of collisions increases when passing from the strict to the statistical implementation, involving more and more participant nucleons and thus more and more neutrons as the latter are more numerous in the target. Conversely, going from the strict to statistical implementation reduces the importance of the protons in the collision process. Note that neutron and proton multiplicities are proportionally less modified when going up in energy. Finally, it is remarkable that the differences between the statistical and strict Pauli blockings concerning multiplicities are rather minute, whatever the incident energy is, at least in the range spanned by table 2 , while, on the other hand, the shapes of the differential cross-section are noticeably different at low energy (see figs. 3 and 15), but very similar to each other at $256 \mathrm{MeV}$, as shown in refs. [2] and [37].

An often debated issue is related to how phase space is populated: can some part of this space be populated only by early emitted particles? This question is partly answered, for our model, by fig. 18. The latter shows the splitting of the energy spectra in contributions coming from emitted particles with different activation numbers. As expected, the high-energy part of the spectra is overwhelmingly dominated by the $n_{\mathrm{a}}=1$ particles. Let us recall that these particles are emitted after the first collision. The top panel of fig. 18 shows that they are more numerous at low than at high outgoing energy. This can be interpreted as follows: in the first collision, the most energetic particle has more chance to re-interact than its partner, simply because the possible collisions of the slowest particle are more efficiently blocked by the Pauli principle. At small angle (central panel of fig. 18), $n_{\mathrm{a}}=1$ particles are mainly emitted with large energy. This can easily be understood by the kinematics of quasi-free scattering. Let us forget Fermi motion for a moment. The first collision is elastic at this incident energy and generates two nucleons emitted in perpendicular directions. The most forward nucleon is the most energetic one. Thus a narrow peak is expected on the high-energy part of the nucleon spectrum. The low-energy side should be very much depleted, since the low-energy particle produced in the elastic collision would always be emitted at larger angle. However, this particle may be reflected before being emitted at a small angle. This is responsible for the small component of the energy spectrum of $n_{\mathrm{a}}=1$ particles emitted at $10^{\circ}$. Fermi motion broadens the high-energy component considerably. At large angles (above $\sim 10^{\circ}$ ), after the first collision, the two particles are emitted with about the same energy (if Fermi motion is disregarded) and at $69^{\circ}$ the situation is largely reversed.

The $n_{\mathrm{a}}=2$ component of the energy spectra is globally as large as the $n_{\mathrm{a}}=1$ component, and peaks at small energy (almost irrespective of the emission angle). The importance of the other components decreases rapidly as $n_{\mathrm{a}}$ increases. Furthermore, the shapes of these components
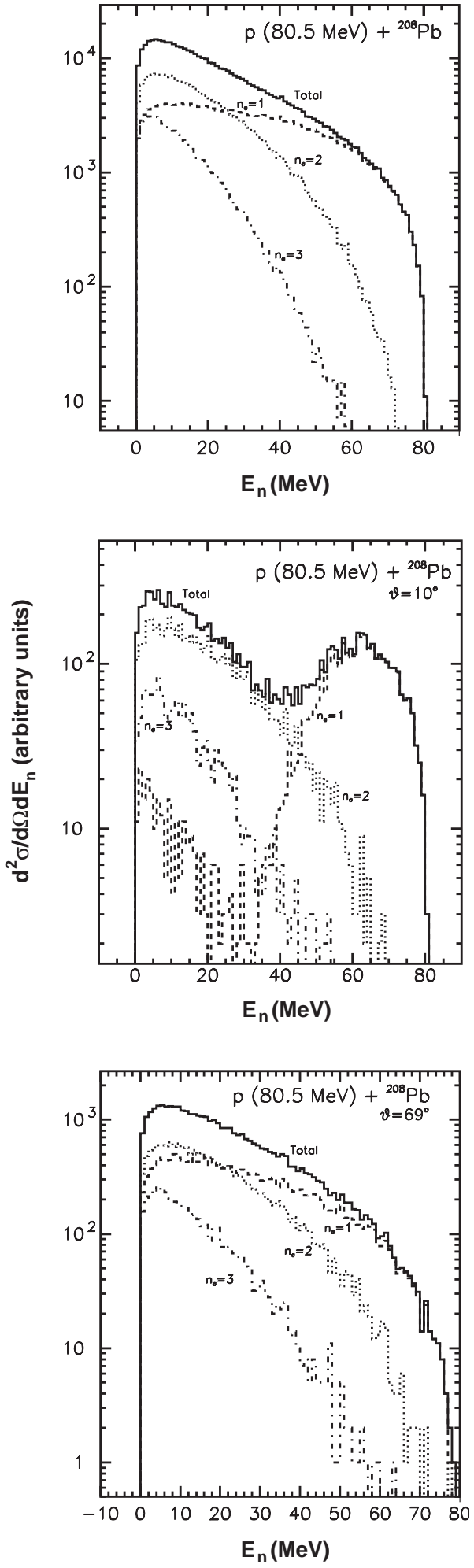

Fig. 18. Splitting of the energy spectra according to the activation numbers of the emitted neutrons. The system under consideration is $p(80.5 \mathrm{MeV})+{ }^{208} \mathrm{~Pb}$. The distributions are given in arbitrary values. The top panel displays the integrated (over angles) energy spectrum, whereas the central and bottom panels refer to emission at $10^{\circ}$ and at $69^{\circ}$, respectively. See the text for details. 

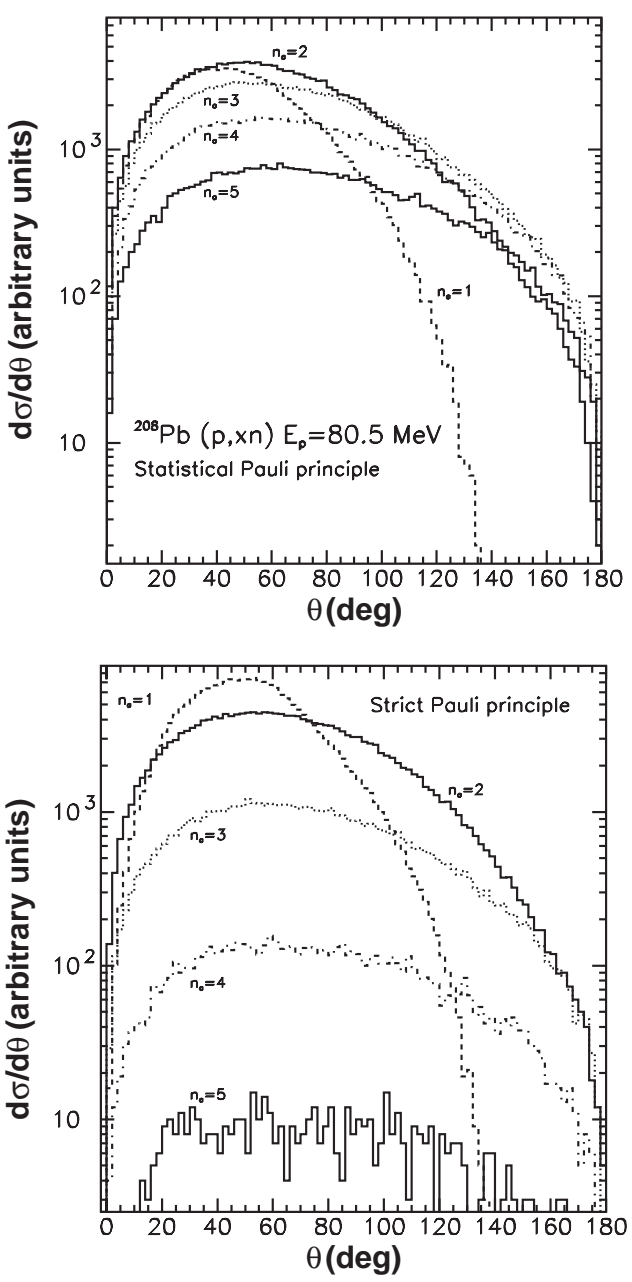

Fig. 19. Splitting of the angular distribution of the emitted neutrons, according to their activation number. The system under consideration is $p(80.5 \mathrm{MeV})+{ }^{208} \mathrm{~Pb}$. The distributions are given in arbitrary values. The top and bottom panels refer to the statistical and strict implementations of the Pauli blocking, respectively.

are very similar to each other and close to the shape of the $n_{\mathrm{a}}=2$ component.

The comparison between the central panel of fig. 18 and fig. 14 reveals that at very small angles, either the theoretical $n_{\mathrm{a}}=1$ component or the $n_{\mathrm{a}}=2$ one (or both) does not have the appropriate shape for the physical case of fig. 14. This might indicate that the target momentum distribution is not realistic in our model or that some quantum interferences manifest themselves in this kinematical region.

Another look at the decomposition in various $n_{\mathrm{a}}$ components is provided by fig. 19, which focuses on the angular distribution of the emitted particles. The $n_{\mathrm{a}}=1 \mathrm{com}-$ ponent has a maximum around $50^{\circ}\left(40^{\circ}\right)$ for the strict (statistical) implementation of the Pauli blocking ${ }^{3}$. The

\footnotetext{
${ }^{3}$ We are just interested here in the relative importance of the components, but in the $\mathrm{d} \sigma / \mathrm{d} \Omega$ distribution, the maximum of the $n_{\mathrm{a}}=1$ component occurs at $33^{\circ}$ and $15^{\circ}$, respectively.
}

other components are much broader and tend progressively toward an isotropic $(\propto \sin \theta)$ pattern. The importance of the successive components decreases with increasing $n_{\mathrm{a}}$ much faster for the strict Pauli blocking than for the statistical one, in agreement with fig. 17. It is remarkable that emission at very forward angles is dominated by the $n_{\mathrm{a}}=2$ component (a little more so for the strict Pauli blocking). Emission at large backward angles is dominated by the $n_{\mathrm{a}}=2$ and 3 components for the strict Pauli principle and by the $n_{\mathrm{a}}=3$ and 4 components for the statistical one. We have checked that emission of fast backward particles (with more than $\sim 40 \mathrm{MeV}$ kinetic energy) requires at least 3 collisions on the average, irrespective of the implementation of the Pauli blocking, but the probability for this emission is much decreased with the strict Pauli blocking, as we already said.

\subsection{Comparison with other models}

Several models have been proposed for nucleon-induced reactions in the energy range $(\sim 40 \mathrm{MeV}-200 \mathrm{MeV})$ under consideration in this paper. The most important ones are the Harp-Miller-Berne model [38], the Griffin exciton model [39-41,43] (in its various versions) and the Feshbach-Kerman-Koonin theory [44]. A review of these models can be found in ref. [45]. The common feature of these models is that the nucleus is viewed as a Fermi gas in a constant average potential well, which can be excited through the creation of particle-hole pairs by the successive nucleon-nucleon collisions and de-excited by emission of particles. The models differ mainly by the sophistication of their respective description of the transition probabilities.

We will not compare here with modern sophisticated models, like the BUU/VUU/BNV model (see refs. [46,47] for a review), mainly because the latter have not been very much tested for nucleon-nucleus collisions, at least at low energy. In addition, these models are numerically much more time-consuming, because of their dynamical treatment of the nuclear mean field. This aspect is of uttermost importance in heavy-ion physics, to which these models are usually applied, but of minor importance in nucleon-nucleus collisions at low energy. Furthermore, the INC model is more suited to handle exclusive observables, like the distribution of particle multiplicities. Therefore the INC model keeps its attractiveness, especially for intensive applications to particle transport calculations.

Many calculations have been made with the Hybrid $(\mathrm{H})$ and Geometry-Dependent Hybrid (GDH) exciton models. Roughly speaking, in these models, the incident particle creates particle-hole pairs (excitons) from which particles can be emitted to the continuum. The energy spectra are then determined by the transition probability to go from the $n$-exciton states to the $(n+2)$-exciton states and by the probability to emit a particle from the $n$-exciton states. The probability for the first process is supposed to be a function of the densities of these respective states. The probability to emit a particle from the $n$ exciton states is generally calculated from the transition 
state method. The expression for the (energy) differential cross-section can be written as [40]

$$
\frac{\mathrm{d} \sigma}{\mathrm{d} \varepsilon}=\sigma_{\mathrm{R}} \sum_{\left[n=n_{0}\right]}^{\bar{n}} \frac{\tilde{\rho}_{n}\left(E^{*}, \varepsilon\right)}{\rho_{n}\left(E^{*}\right)} \frac{\lambda_{c}(\varepsilon)}{\lambda_{c}(\varepsilon)+\lambda_{+}} D_{n}
$$

where $\sigma_{\mathrm{R}}$ is the reaction cross-section, the sum runs over the number of excitons, $\rho_{n}\left(E^{*}\right)$ is the density of $n$-exciton states at excitation energy $E^{*}, \tilde{\rho}_{n}\left(E^{*}, \varepsilon\right)$ is the density of $n$-exciton states at excitation energy $E^{*}$ with an exciton having precisely energy $\varepsilon, \lambda_{c}(\varepsilon) \approx \sigma(\varepsilon) \varepsilon m / \hbar^{2}$ (with $\sigma(\varepsilon)$ being the inverse capture cross-section for a nucleon of energy $\varepsilon$ ) is related to the emission rate into the continuum of particles with energy $\varepsilon, \lambda_{+}$is the nucleon mean free path for generating in the target a transition from $n$-exciton to $(n+2)$-exciton states and where $D_{n}$ is the survival probability of the $n$ exciton states (see ref. [40] for details). In the $\mathrm{H}$ model, this formula is applied directly, with parameters representing the average conditions, whereas in the GDH model, it is applied for each impact parameter with the conditions $\left(\sigma(\varepsilon), \lambda_{+}\right.$and $\left.D_{n}\right)$ pertaining to the (linear) trajectory of the incident particle for this impact parameter. A summation over the latter is performed. The quantity $\lambda_{+}$is usually calculated in terms of the nucleon-nucleon cross-section, corrected for Pauli blocking according to the Kikuchi-Kawai prescription [51]. It has also been tentatively related to the imaginary part $(W)$ of the nucleon optical-model potential $\left(\lambda_{+} \approx \hbar v / W\right)$, where $v$ is the average velocity. We refer to refs. $[40,41]$ for more details.

We present in the top panel of fig. 3 a comparison of the predictions of the $\mathrm{H}$ and GDH models with our results for a typical case. As often quoted in the literature [20], the GDH model is generally better than the $\mathrm{H}$ model, especially for the high-energy part of the spectrum. This is expected since the corresponding particles are mainly produced in quasi-elastic collisions which are more represented in the peripheral collisions. Figure 3 also shows that our INC model gives a reasonable agreement, similar to the one achieved by the exciton model. We also compared our predictions with the GDH model in figs. 1-2 for neutron energy differential cross-sections in protoninduced reactions. At low energy (fig. 1), the GDH model is definitely better than the INC model, but at $45 \mathrm{MeV}$ (fig. 2), the two models produce similarly reasonable results. The INC model is better than the GDH model for heavy targets whereas the converse is true for light targets. The results of the GDH model used in ref. [17] are evaluated with the two choices mentioned above for the quantity $\lambda_{+}$. The use of the optical model seems slightly better. We also compared our results with a version of the GDH model which is adapted to the prediction of the double differential cross-sections. In short, this version introduces angular distributions which are supposed to characterize the emission pattern of the various exciton states: the free nucleon-nucleon cross-section is taken for the $n=1$ exciton state, a folding of two such distributions is taken for $n=3$, and so on. Often, empirical distributions are used. Such a comparison is provided by fig. 5, where angular distributions for given outgoing energies are displayed. Except for the small angles (less than $\sim 15^{\circ}$ ), the predictions of the INC model are noticeably better than those of the GDH model. The GDH model seems to predict a steep drop in the various angular distributions, which is not observed experimentally. This feature is probably induced by too crude assumptions for the angular distributions pertaining to the emission from the various $n$ exciton states.

In figs. 7-8, we display a comparison with the GDH model for proton emission in proton-induced reactions. At low energy (fig. 7), the energy differential cross-section is definitely better reproduced with the GDH model, but at $62 \mathrm{MeV}$, the difference is less pronounced.

Some authors $[52,42,43,17]$ noticed that the most important contributions come from low exciton numbers. As a consequence, it is reasonable to consider that probabilities to excite these exciton states should not be determined by phase space only, but also by the properties of the nucleon-nucleon collisions. Therefore, angular distributions should be taken as close as possible to those dictated by the successive collisions. Following these considerations, Smith and Bozoian [49] have built a model where the lowest contribution of the GDH model is replaced by a quasi-free scattering (QFS) contribution, calculated microscopically, with some "effective" nucleon number. This model gives better results than the standard GDH model, as shown in fig. 8, except at small angles and high-energy loss, similarly to our INC results. It is also interesting to compare the various contributions of this model and those of ours. They are given in fig. 5 of ref. [49] and fig. 18, respectively. The shape of the QFS contribution is similar to the $n_{\mathrm{a}}=1$ contribution of our model. The shapes of the $3 \mathrm{p}-2 \mathrm{~h}, 4 \mathrm{p}-3 \mathrm{~h}, \ldots$ contributions are rather similar to each other and quite similar to the shapes of the $n_{\mathrm{a}}=2,3, \ldots$ contributions in our model. This more or less confirms that the activation number $n_{\mathrm{a}}$ can be closely related to the exciton number. We have to stress, however, that the relative importance of the QFS contribution of ref. [49] is substantially larger than the $n_{\mathrm{a}}=1$ contribution in our model.

A step further was taken by Chiang and Hüfner [34] (and, independently, by $\mathrm{Wu}$ [53]). They assumed that the double differential cross-section can be decomposed into three terms, corresponding, respectively, to single (1), double (2) scattering and compound-nucleus (c) formation and decay:

$$
\frac{\mathrm{d} \sigma}{\mathrm{d} \Omega \mathrm{d} \varepsilon}=\sum_{\alpha=1,2, c} N_{\alpha} \sigma_{\alpha}\left(\varepsilon_{0}\right) F_{\alpha}\left(\Omega, \varepsilon ; \varepsilon_{0}\right),
$$

where $N_{\alpha}$ gives the number of nucleons after step $\alpha$, the $\sigma_{\alpha}$ 's are the cross-sections for single, double scattering and compound nucleus, the functions $F_{\alpha}$ are the angular distributions for particle emission during the successive steps and where $\varepsilon_{0}$ is the incident energy. The quantities $\sigma_{\alpha}$ are evaluated by simple Glauber formulae. The angular distribution $F_{1}$ is taken from the parametrization of the angular distribution following nucleon-nucleon elastic scattering in nuclear matter, as calculated in ref. [51]. The angular distribution $F_{2}$ is a folding of $F_{1}$ by itself, both 
in angle and energy, and the angular distribution $F_{c}$ is isotropic. In other words, the authors postulate that only two collisions contribute to the pre-equilibrium emission, the third one leading already to the formation of an equilibrated system. Of course, this statement can be true only if the incident energy is low enough. Chiang and Hüfner argue that it holds up to $\sim 100 \mathrm{MeV}$. This conjecture is supported to some extent by fig. 19: the components for $n_{\mathrm{a}} \geq 3$ are indeed close to be isotropic.

A comparison between the predictions of our model and those of Chiang and Hüfner is provided by fig. 9 . They are very similar, although a slightly better agreement with experiment can be noticed for our INC model, especially for large and small angles. This is perhaps not surprizing as the average number of collisions in the cascade stage (see table 2) lies about 2 . It seems that the "one, two, infinity" approach is close to reality. This approach, which was introduced in a pragmatic way, using plausibility arguments about mean free path, energy loss, Pauli blocking, etc., is supported by our model, where all these elements are treated equally by the INC dynamics.

In more recent years, attempts have been made to describe the nuclear reactions with more elaborated tools, derived from the Feshbach-Kerman-Koonin theory. For the energy range under interest here, the multi-step direct (MSD) model is the most appropriate one. We refer to ref. [48] for a description of the method. Let us just say here that it can be viewed as an exciton model with an elaborate way to calculate the transition probabilities, based on some statistical DWBA methods. Figure 3 shows the results of such a calculation (for three angles). They give a near perfect agreement, owing to a fit of the interaction strength and to some assumptions about the (average) spectroscopic factors. Furthermore, it seems that this strength should be adjusted differently when changing the incident energy. It is gratifying to see that a simple model like our INC model can yield results of comparable (but definitely slightly lower) quality as these elaborated approaches.

Recently, a very complex tool for calculating the nuclear cross-sections, namely the TALYS code system, has been built [35]. It incorporates many models (Hauser-Feshbach, compound-nucleus, MSD, DWBA to discrete low-lying states and to giant resonances, etc.). An example of the results provided by this elaborate model is illustrated by fig. 12. As expected, these results are much better than ours, but one has to keep in mind that some amount of parameter fitting allows this kind of agreement. It should be noticed that our INC model reproduces the general trends of the cross-sections, in spite of its simplicity.

In summary, the INC model produces quite reasonable results in view of its simplicity, of its lack of free parameters and of the poor fulfillment of the theoretical conditions of validity (eq. (2)). The predictions seem to be better when the incident energy increases, when the target mass increases and for angles that are intermediate. Nonetheless, our model can compete reasonably well with most other popular models.

\subsection{Possible improvements}

This last remark opens the prospect of future improvements of our model in the $40-200 \mathrm{MeV}$ range. Two features have been touched upon in this paper. The introduction of a "mixed" Pauli blocking, perhaps hard to justify theoretically, can anyway be quite efficient, as we have indicated. Another possibility is provided by the introduction of in-medium elementary cross-sections, which seems to improve the results of the GDH model. Another possible improvement would consist in introducing more realistic momentum distributions. We have also investigated several other modifications, like the modification of the angular distribution in nucleon-nucleon elastic scattering, the introduction of refraction for particles leaving the nuclear volume, the variation of the potential depth. Results will be published elsewhere [54], but none of them brings substantial improvement. Finally, we mention that a feature lacking in our approach is the production of composites, which is experimentally important in this energy range, unlike what happens at higher incident energy.

\section{Conclusion}

We have investigated the low-energy limit of validity of the INC model by comparing the predictions of the Liège model (INCL3 version) to experimental data and to predictions by other models. Contrary to what is generally believed, the predictions of our INC model are often reasonably good, well below the commonly accepted limit of validity, i.e. $\sim 250 \mathrm{MeV}$ incident energy. There are however systematic variations of the agreement as explained in sect. 3 . We have seen also that the predictions of our model are comparable with and sometimes better than those of the exciton model or of its more elaborated versions like the GDH model and even those of the MSD model. This may not be after all so surprising: all these models carry the same basic physics, i.e. the physics of a Fermi gas lying in a potential well and subject to excitation by collisions and de-excitation by emission of particles. Only the methods for evaluating the different transition probabilities for the various steps of this complex process are different. The INC model presents this advantage that all transition probabilities are automatically fixed by the handling of collisions, giving it the unity which is somehow lacking in other models, where different methods are used for evaluating the various probabilities. The remaining main difference in the physics between the INC model and the other ones is the fact that the single-particle spectrum is continuous in the INC model, whereas it is discrete in the other models. This is perhaps the reason why the INC model has been discarded for the low-energy domain, in spite of earlier results obtained by some authors [55]. Our results seem to show that the discrete nature of the singleparticle spectrum has no obvious effect. A possible explanation may lie in the fact that in reality, single-particle excitations in nuclei have some width, which may wash out the discrete structure [56]. 
We want to recall that we use the INC model for describing the dynamics in the impact parameter range. We do not use it for the total reaction cross-section (in fact our predictions for this quantity are too small, by a factor between one and two). This is the way INC codes are often used in transport codes.

The agreement that we obtain in this work would allow our model to be introduced in such transport codes for a large domain of energy. This is an important point: the simplicity of the INC model and the subsequent fastness of the associated numerical codes make it particularly appropriate for the modeling of nucleon-nucleus reactions in extensive applications. This plainly justifies the present investigation. The accuracy of our results is admittedly not perfect and may sometimes be considered as insufficient, depending upon the application of the model. That is why we do not provide a definite figure for the lower limit of validity of the INC. But for transport codes which have to handle a series of complex processes with many different energy scales, the accuracy reached by the INC model in the $40-200 \mathrm{MeV}$ range is very promising and the perspective of having a simple model in a large energy domain is certainly an attractive one.

This work is a participation in the HINDAS collaboration (European Union Contract No. FIKW-CT-2000-00031). We acknowledge the EU financial support. We are grateful to our HINDAS colleagues for interesting discussions.

\section{References}

1. J. Cugnon, Nucl. Phys. A 389, 191c (1982).

2. J. Cugnon, C. Volant, S. Vuillier, Nucl. Phys. A 625, 729 (1997).

3. J. Cugnon, J. Vandermeulen, Ann. Phys. (Paris) 14, 49 (1989).

4. R. Serber, Phys. Rev. 72, 1114 (1947).

5. G. Bertsch, Prog. Part. Nucl. Phys. 4, 483 (1980).

6. S. Ayik, C. Grégoire, Phys. Lett. B 212, 269 (1988).

7. W. Botermans, R. Malfliet, Phys. Lett. B 215, 617 (1988).

8. R. Malfliet, Nucl. Phys. A 545, 3c (1992).

9. V.E. Bunakov, G.V. Matvejev, Z. Phys. A 322, 511 (1985).

10. P. Pawłowski et al., Eur. Phys. J. A 9, 371 (2000).

11. D. Doré et al., Phys. Rev. C 63, 034612-1 (2001).

12. L. Dresner, Oak Ridge report ORNL-TM-196 (1962).

13. G.S. Bauer, 2nd International Conference on Accelerator Driven Transmutation Technologies and Applications, edited by H. Condé (Gotab Publisher, Stockholm, 1997).

14. R.E. Prael, H. Liechtenstein, report LA-UR-89-3014, Los Alamos National Laboratory (1989).

15. J. Cugnon, P. Henrotte, Proceedings of the 5th Workshop on Simulating Accelerator Radiation Environments SARE5, edited by D. Filges, F. Goldenbaum, Y. Yariv, Jülich publication ESS112-10-T (2001) p. 65.
16. W. Scobel, M. Blann, T.T. Komoto, S.M. Grimes, L.F. Hansen, C. Wong, B.A. Pohl, Phys. Rev. C 30, 1480 (1984).

17. M. Blann et al., Nucl. Phys. A 257, 15 (1976).

18. F.E. Bertrand, R.W. Peelle, Phys. Rev. C 8, 1045 (1973).

19. N. Marie et al., Measurements of neutron production double differential cross-section in 62.5 MeV proton-induced reactions, University of Caen preprint (2002).

20. M. Trabandt et al., Phys. Rev. C 39, 452 (1989).

21. J.R. Wu, C.C. Chang, H.D. Holmgren, Phys. Rev. C 19, 698 (1979).

22. R.E. Segel et al., Phys. Rev. C 26, 2424 (1982).

23. W.B. Amian et al., Nucl. Sci. Eng. 102, 310 (1989).

24. S.V. Förtsch et al., Phys. Rev. C 43, 691 (1991).

25. S. Scobel et al., Phys. Rev. C 41, 2010 (1990).

26. A.A. Cowley et al., Z. Phys. A 336, 189 (1990).

27. H. Machner et al., Phys. Lett. B 138, 39 (1984).

28. S. Stamer et al., Phys. Rev. C 47, 1647 (1993).

29. A. Koning et al., HINDAS - A European Nuclear Data Program for Accelerator-Driven Systems, J. Nucl. Sci. Technol. Suppl. 2, 1161 (2002).

30. D. Hermsdorf et al., Dresden Zfk-277 report (1974).

31. E.L. Hjort et al., Phys. Rev. C 53, 237 (1996).

32. A. Ringbom et al., Nucl. Phys. A 617, 316 (1997).

33. H.P. Wellish, D. Axen, Phys. Rev. C 54, 1329 (1996).

34. H.C. Chiang, J. Hüfner, Nucl. Phys. A 349, 466 (1980).

35. A. Koning, S. Hilaire, in preparation.

36. J.P. Meulders, High and Intermediate Energy Nuclear Data for Accelerator-Driven Systems, European Commission report P\&T-HINDAS-S1 (2001).

37. S. Vuillier, PhD Thesis, University of Orsay (1998).

38. G.D. Harp, J.M. Miller, B.J. Berne, Phys. Rev. 165, 1166 (1968).

39. J.J. Griffin, Phys. Rev. Lett. 17, 478 (1966).

40. M. Blann, Phys. Rev. Lett. 27, 337, E700 and E1550 (1971).

41. M. Blann, Phys. Rev. Lett. 28, 757 (1972).

42. M. Blann, Nucl. Phys. A 213, 570 (1973).

43. M. Blann, Annu. Rev. Nucl. Sci. 25, 123 (1975).

44. H. Feshbach, A. Kerman, S.E. Koonin, Ann. Phys. (N.Y.) 125, 429 (1980).

45. H. Gruppelaer, P. Nagel, P.E. Hodgson, Riv. Nuovo Cimento 9, No. 7 (1986).

46. G.F. Bertsch, S. Das Gupta, Phys. Rep. 160, 189 (1988).

47. A. Bonasera, F. Gulminelli, J. Molitoris, Phys. Rep. 243, 1 (1994).

48. R. Bonetti, D. Camnasio, L. Colli Milazzo, P.E. Hodgson, Phys. Rev. C 24, 71 (1981).

49. R.D. Smith, M. Bozoian, Phys. Rev. C 39, 1751 (1989).

50. H.H. K. Tang, G.R. Srinivasan, N. Azziz, Phys. Rev. C 42, 1598 (1990).

51. K. Kikuchi, M. Kawai, Nuclear Matter and Nuclear Reactions (North-Holland, Amsterdam, 1968).

52. M. Blann, F.M. Lanzafame, Nucl. Phys. A 142, 559 (1970).

53. J.R. Wu, Phys. Lett. B 91, 169 (1980).

54. P. Henrotte, in preparation.

55. H.W. Bertini, Phys. Rev. C 5, 2118 (1972).

56. C. Mahaux, R. Sartor, Adv. Nucl. Phys. 20, 1 (1991). 